\title{
Development of a Setup for Material Identification Based on Laser-Driven Neutron Resonance Spectroscopy
}

\author{
Marc Zimmer ${ }^{1 *}$, Stefan Scheuren ${ }^{1}$, Annika Kleinschmidt ${ }^{2,3}$, Alexandra Tebartz ${ }^{1}$, Tina Ebert $^{1}$, Johannes Ding ${ }^{1}$, Daniel \\ Hartnagel $^{1}$, and Markus Roth $^{1}$ \\ ${ }^{1}$ Technische Universität Darmstadt, Institut für Kernphysik, 64289 Darmstadt, Germany \\ ${ }^{2}$ GSI Helmholtzzentrum für Schwerionenforschung, 64291 Darmstadt, Germany \\ ${ }^{3}$ Helmholtzinstitut Jena, 07743 Jena, Germany
}

\begin{abstract}
With the phasing out of many research reactors over the upcoming years, a shortcoming of small and medium sized neutron sources is to be expected. Laser-driven neutron sources have the potential to fill this void, with enormous progress being made in laser technology over the past years. Upcoming petawatt lasers with high repetition rates up to $10 \mathrm{~Hz}$ promise a tremendous increase in neutron flux. In this paper, a setup is developed and optimized to conduct neutron resonance spectroscopy at a laser-driven neutron source. This setup is then evaluated at an experimental campaign at the PHELIX laser system. Laser intensities up to $10^{21} \mathrm{~W} / \mathrm{cm}^{2}$ with a ns pre-pulse contrast of $10^{-7}$ were used for ion acceleration, resulting in $(1.8 \pm 0.7) \times 10^{8} \mathrm{~N} / \mathrm{sr}$ per pulse corresponding to $(2.3 \pm 1.0) \times 10^{9} \mathrm{~N}$ in a $4 \pi$ equivalent. These pulses were moderated, collimated and investigated via the time of flight method in order to characterize the thermal neutron spectrum as well as the signal to noise ratio.
\end{abstract}

\section{Introduction}

Neutrons provide a unique tool for material analysis, from neutron diffraction [1] over prompt gamma neutron activation analysis [2] to Neutron Resonance Spectroscopy (NRS) [3]. These techniques can be used to identify the elemental and isotopic compositions of unknown materials without compromising the structural integrity of the sample. A key feature of neutrons is the large propagation range into materials. In combination with the unique distribution of scatter and capture resonances for every isotope, neutrons provide a particularly valuable analysis tool, complementing currently existing $\mathrm{x}$-ray techniques.

In previous years, neutrons were in many cases provided by research reactors. With recent developments, a large fraction of these sources have been or will be shut down in the near future and a need for small and medium-sized neutron sources is growing [4]. Laser-Driven Neutron Sources (LDNS) promise to fulfill these needs and provide a compact short pulsed neutron source with high peak flux [5]. Recent improvements in laser technology enable high-intensity lasers to be operated up to $10 \mathrm{~Hz}$ [6], therefore, tremendously increasing the average neutron flux of these sources.

LDNS are especially well suited for material analysis utilizing NRS since it relies on the Time of Flight (ToF) method which requires short initial pulse durations to decrease the energy uncertainty. LDNS have sub-ns production pulse lengths and consequently the dominant energy uncertainty is the moderation time which is below $2 \mu \mathrm{s}$ for neutrons above $1 \mathrm{eV}$. Other Compact Accelerator Driven Neutron Sources (CANS) or reactors have longer pulse lengths above $100 \mu \mathrm{s}$ [7] and therefore require longer flight paths to maintain the same energy uncertainty [3].

Spallation sources can operate similarly to LDNS in a regime only limited by the moderation time but they need longer flight paths due to the larger shielding required for higher neutron energies from the spallation process [8]. A longer flight path $L$ results in a reduction in neutron flux at the detector since the flux decreases with $1 / L^{2}$. This enables a more efficient utilization of the neutron beam in comparison to most other sources, except those based on electron linacs which can reach similar short pulse lengths and neutron energies.

NRS exploits the unique resonance distribution for most isotopes in their neutron cross section $\sigma(E)$. Therefore, when a neutron pulse with a broad energy spectrum, as it is produced by LDNS, travels through a sample, resonances with a high cross section will absorb parts of the spectrum, imprinting this information on the neutron distribution. A detector behind the sample can, therefore, identify the presence and the amount of each isotope in relation to the depth and position of the absorption peaks. The following part of this publication will describe the optimization and the characterization of an LDNS for NRS.

\footnotetext{
* Corresponding author: $\underline{\text { m.zimmer@gsi.de }}$
} 


\section{Setup}

For this experiment, the PHELIX laser at GSI was used. The $(600 \pm 100)$ fs laser pulse [9] was focused to a FWHM diameter of $(4 \pm 0.5) \mu \mathrm{m}$ with $(180 \pm 12) \mathrm{J}$ per pulse. This setup allows peak intensities of up to $10^{21} \mathrm{~W} / \mathrm{cm}^{2}$ enabling ion acceleration via the Target Normal Sheath Acceleration (TNSA) [10] and via relativistic induced transparency that can commonly deliver ion energies between 60 and $85 \mathrm{MeV}$ [11]. During this campaign, the ns laser contrast was reduced to $10^{-7}$ and consequently, maximum ion energies were limited to around $(33 \pm 10) \mathrm{MeV}$. As target material, deuterated polystyrene foils with $500 \mathrm{~nm}$ thickness as well as 10, 25 and $50 \mu \mathrm{m}$ silicon targets were used. Some of them with a micro-needle structure on the laser facing surface and a flat rear side for better conversion efficiency.

The neutron production is carried out using a pitchercatcher design [5] where ions are directed from the target (pitcher) onto a beryllium cylinder (catcher). There, the ions are converted into neutrons via ${ }^{9} \mathrm{Be}(\mathrm{d}, \mathrm{n}){ }^{10} \mathrm{~B}$, ${ }^{9} \mathrm{Be}(\mathrm{p}, \mathrm{n}){ }^{9} \mathrm{~B}$ as well as stripping reactions. Emitted neutrons have energies in the $\mathrm{MeV}$ regime, but for most NRS applications, neutrons in the epithermal ( $\sim 1$ to 100's of $\mathrm{eV}$ ) regime are required. Therefore, the neutrons are directed into a moderator made out of High Density PolyEthylene (HDPE). The high hydrogen content slows neutrons down until they are emitted via diffusion at the moderator surface and propagate towards the detector.

To ensure only neutrons with a direct flight path from the moderator to the detector are counted, a series of borated HDPE plates are installed. These plates have an opening in the center with a converging diameter towards a borated Multi-Channel Plate (MCP) detector to act as a collimator as seen in Fig. 1.

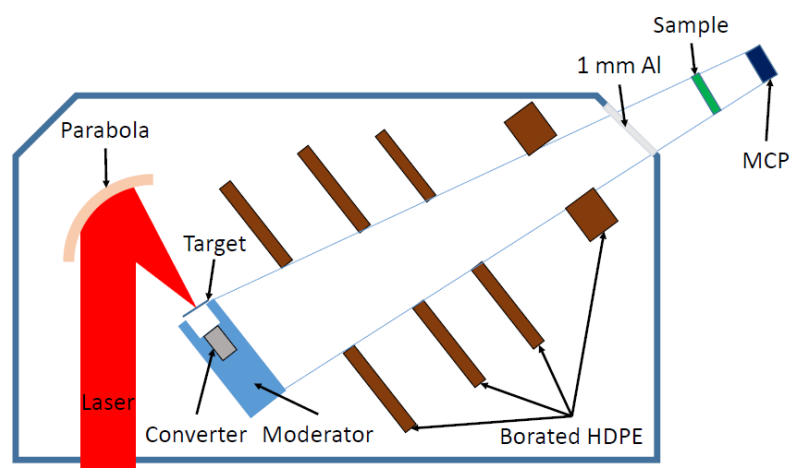

Fig. 1. Setup of the experiment. Neutrons are produced inside the converter, slowed down in the moderator and are emitted to the MCP via the flight path indicate by the light-blue lines.

The sample is placed at $10 \mathrm{~cm}$ distance in front of a MCP that is capable of single neutron detection. In this configuration, absorption gamma photons produced inside the sample have a smaller chance of reaching the detector in comparison to placing the sample directly in front of the detector.
The MCP itself is placed at a distance of $(180 \pm 1) \mathrm{cm}$ and is additionally shielded with $10 \mathrm{~cm}$ borated HDPE in all other directions which is not shown in Fig. 1 to improve clarity. The detector used in this experiment is a custom made design, consisting of a borated MCP from Nova Scientific encapsulated in a vacuum housing by the ProxiVision $\mathrm{GmbH}$ with a spatially integrated anode for a faster time response. This detector is capable of single neutron detection with a maximal time resolution of 10 ns. The neutron energy was calculated via ToF. The neutron flight time $t$ was determined by:

$$
t=t_{d}-t_{0}-t_{c}-t_{s}
$$

With the detection time $t_{d}$, the arrival time of the gamma flash $t_{0}$, the time delay of light to propagate from the target to the detector $t_{c}=6 \mathrm{~ns}$ and the average delay neutrons of a certain energy need to cross the surface of the moderator $t_{s}$.

\section{Design and Simulations}

\subsection{Catcher}

During the operation of an LDNS, the beryllium catcher has to be shielded against ablation by the expanding hot laser plasma, since beryllium dust is highly toxic and the integrity of the catcher has to be maintained. Therefore, a blast shield in front of the beryllium is required. In previous experiments [12], a $1 \mathrm{~mm} \mathrm{Al}$ plate was used. Inside this blast shield deuterons with an energy below 18 $\mathrm{MeV}$ are stopped and do not reach the converter. Al itself has a low neutron yield and consequently, a large amount of ions does not contribute to the neutron production. Hence a material with low stopping power, high thermal and ablation resistivity, as well as relatively high neutron yield from deuteron bombardment should be chosen as a blast shield. Cirlex consists of a polyimide material with a high thermal resistivity and has a high carbon content which has a relatively high $(\mathrm{d}, \mathrm{n})$ cross section of $200 \mathrm{mb}$ between 2 and $10 \mathrm{MeV}$ [13]. It is comprised of only low $\mathrm{Z}$ materials, resulting in a low stopping power thus more deuterons $(>14 \mathrm{MeV})$ arrive at the catcher. Therefore, it fulfills the requirements as laser-plasma blast shield. In addition, the reaction products of $\mathrm{d} / \mathrm{p}$ irradiation produce mostly short-lived radioactive isotopes like ${ }^{13} \mathrm{~N}$ with a half-life of 10 minutes, ${ }^{11} \mathrm{C}$ with 20 minutes and stable ${ }^{14} \mathrm{~N}$. This reduces the radiation inside the chamber and enables fast target handling.

\subsection{Moderator}

Designing a moderator for an epithermal neutron source is fundamentally different from thermal or cold neutron sources. While these sources use reflectors to keep neutrons inside the moderator as long as possible until they diffuse out at the surface [8], an epithermal source has to emit neutrons before they reach thermal equilibrium. On average, neutrons undergo 18 collisions inside HDPE until they are thermalized. For $1 \mathrm{eV}$ they have to be emitted after around 16 collisions. This can be 
achieved when an optimal thickness is found, where the average scattering number at the surface is corresponding to the desired energy for NRS. To find an optimal moderator configuration, a PHITS [14] simulation was used to scan the length height and width of the moderator while measuring the epithermal neutron flux at a distance of $50 \mathrm{~cm}$ to the moderator center. The neutron spectrum was produced by directing a TNSA ion spectrum with up to $32 \mathrm{MeV}$ protons on to a beryllium catcher. Fig. 2 shows the results for the length and the height. It can be seen, that for both cases a saturation effect starts to appear for values larger than $10 \mathrm{~cm}$. The preferred dimensions of this cuboid moderator were chosen to be at a length of $17 \mathrm{~cm}$ and a height of $10 \mathrm{~cm}$ towards the center.
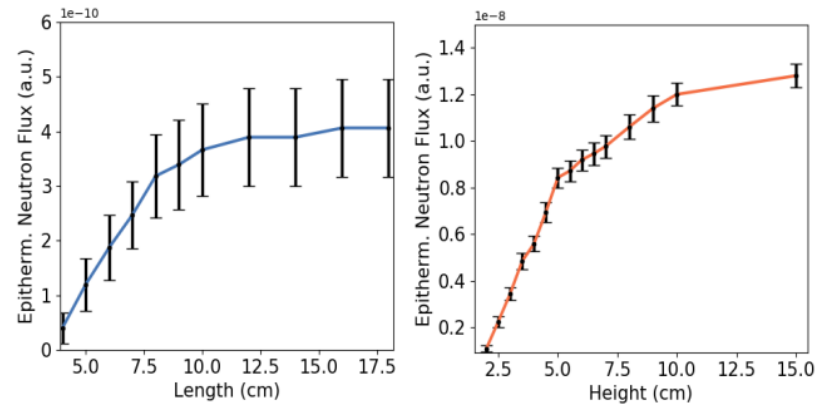

Fig. 2. Epithermal neutron flux as a function of moderator length and height. For both parameters a saturation effect is visible. Simulation performed with the PHITS code.

Fig. 3 shows the increase in epi-thermal flux as a function of moderator width. The flux increases with the thickness until a maximum of $4 \mathrm{~cm}$ towards the moderator center is reached. Afterwards the flux decreases until a width of 5 $\mathrm{cm}$ where the flux increases again until it reaches a second maximum at $5.5 \mathrm{~cm}$. Further increasing the width causes the flux to decrease. The second peak is associated with neutrons that have an initial propagation direction towards the moderator surface as these neutrons pass through less material as those emitted in other directions.

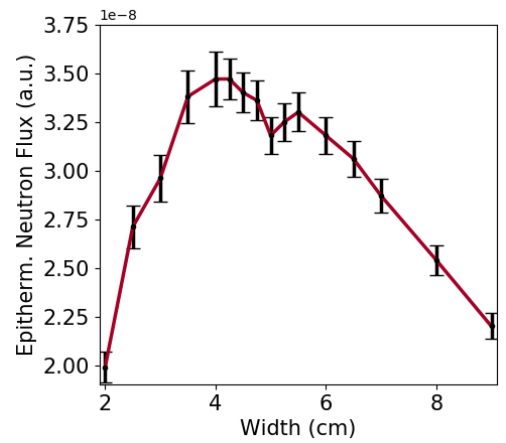

Fig. 3. Epithermal neutron flux as a function of moderator width. First, the flux is increasing with the moderator width until it reaches a maximum at $4 \mathrm{~cm}$. Afterwards, the flux decreases until $5 \mathrm{~cm}$ where it starts increasing again. At $5.5 \mathrm{~cm}$ a second maximum is visible followed by a constant decrease in neutron flux. Simulation performed with the PHITS code.

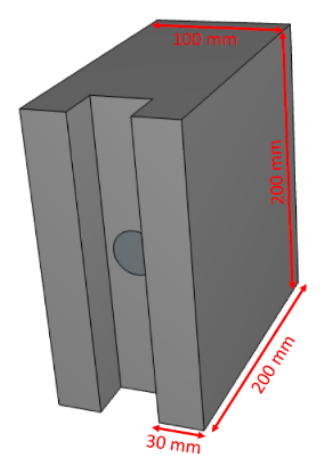

Fig. 4. Optimized moderator design for NRS. The dark circle in the middle represents the Be catcher. In the front the wings are visible.

In addition, two $3 \times 3 \times 20 \mathrm{~cm}$ wings were added in front of the moderator as seen in Fig. 4. These wings have a chance to capture neutrons that are emitted into laser backwards direction up to an angle of $56^{\circ}$ measured from the converter front surface center. PHITS simulations have shown, that this can lead to an increase in epithermal neutron flux at the detector facing moderator side of $72 \%$ and an increase of thermal flux of $46 \%$ as seen in Fig. 5. The presence of the wings leads to a new optimal moderator thickness of $5 \mathrm{~cm}$. Fig. 4 shows a CAD drawing of the optimized moderator design.

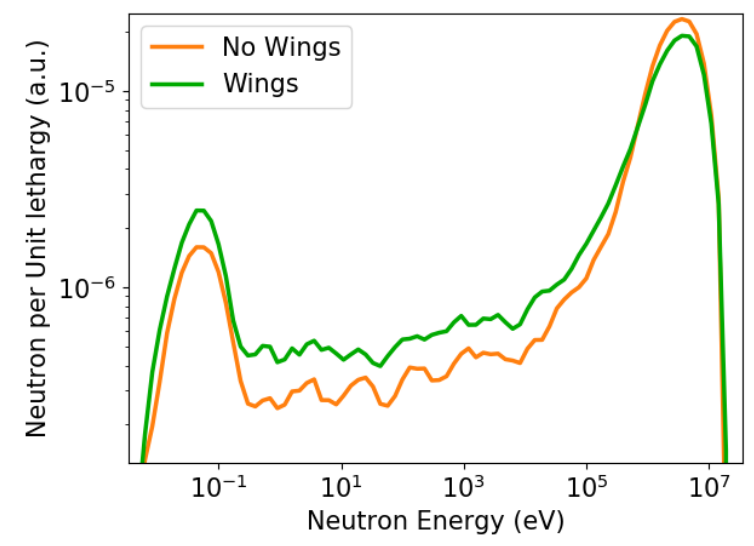

Fig. 5. PHITS simulation for the moderator with and without moderation wings. The epithermal yield is increased by $72 \%$ as well as the thermal yield by $46 \%$

With a PHITS simulation, the flux of neutrons between 1 and $10 \mathrm{eV}$ is investigated at the detector facing moderator surface. The result can be seen in Fig.6. Most neutrons in this energy regime are emitted in close proximity to the converter, indicated by the black square. 


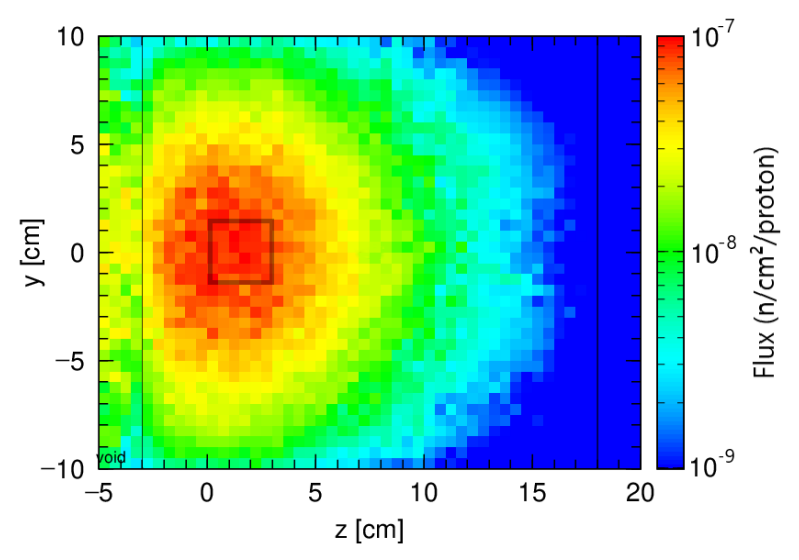

Fig. 6. Flux of neutrons with energies between 1 and $10 \mathrm{eV}$ at the moderator surface. Most neutrons are emitted close to the catcher, indicated by the black square.

The energy resolution of an NRS measurement and, therefore, the minimal detector distance is limited by the uncertainty of the arrival time $\Delta t$. There are two main contributing factors to this uncertainty for an LDNS. The first one is the moderation time, e.g. the time a neutron propagates inside the moderator until it reaches its final energy. The second one is the time after the last collision until the neutron reaches the moderator surface. The sum of these two factors is estimated in a PHITS simulation for this moderator design for various energies from $1 \mathrm{eV}$ to $1 \mathrm{keV}$. The results can be seen in Fig. 7. It is visible that for higher energies the average arrival time shifts to earlier times. Also, the width of each pulse is decreasing with higher energies. The average arrival time can be treated as an offset in the energy determination, while the width of each peak represents the uncertainty for each energy $\Delta t_{s}(E)$. For $1 \mathrm{eV}$ this width is $(2 \pm 0.5) \mu \mathrm{s}$, for 100 $\mathrm{eV}(200 \pm 50) \mathrm{ns}$ and for $1 \mathrm{keV}$ this width has decreased to $(60 \pm 10) \mathrm{ns}$. If this is compared to the minimal ion pulse width at the LANSCE spallation source of $125 \mathrm{~ns}$ [8] convoluted with their moderation time, it becomes evident that an LDNS has the potential to achieve a higher energy resolution for neutron energies above $100 \mathrm{eV}$.

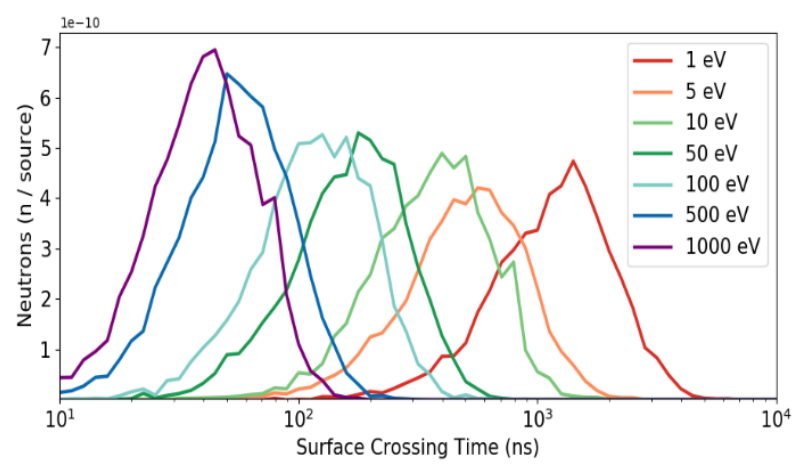

Fig. 7. Time needed for neutrons with different energies to cross the moderator surface. Faster neutrons leave the moderator quicker and have a lower moderation time uncertainty. Energy intervals are $\pm 1 \%$.

\subsection{Collimation System}

For NRS, it is crucial that neutrons detected at the MCP have traveled in a straight line from the moderator through the sample. Therefore, neutrons have to be prevented from scattering at other parts of the experimental setup, the 3 $\mathrm{cm}$ thick steel target chamber wall, the floor or the ceiling. Therefore, three $5 \mathrm{~cm}$ thick and $50 \mathrm{~cm}$ wide borated HDPE sheets were placed in the beam path with an inner recess fitting the converging projection of the moderator onto the MCP as seen in Fig. 1 and 8. In addition to the three large collimators, four smaller collimators with converging round openings to the detector were used. Two of them before the $1 \mathrm{~mm}$ aluminium flange inside the target chamber and two additional collimators directly in front of the detector. This geometry enables to view the entire moderator from the detector to increase the amount of usable neutrons. The increase in energy uncertainty for this setup from the larger visible surface area is below $1 \%$ (at $4 \mathrm{eV}$ ). This is smaller than the corresponding uncertainty from $\Delta t_{s}$ with $3 \%$.

The detector itself was surrounded by a $1 \mathrm{~mm}$ copper Faraday cage against the EMP and additional $10 \mathrm{~cm}$ borated HDPE in all other directions. The setup was reconstructed in a PHITS simulation to evaluate the collimation and the signal to noise ratio. The result can be seen in Fig. 8 for neutrons with energies between 1 and 10 $\mathrm{eV}$. The collimator achieves, in this simulation, a signal to background ratio of $(15 \pm 4): 1$ inside the detector shielding. An analysis of the arrival time and the energy of the neutrons revealed the origin of the background neutrons to be from fast neutrons that have been moderated in the last borated HDPE layer. Additional contributions to the background have been identified to come from the prompt gamma emission from neutron absorption in boron, hydrogen and carbon. This background can be significantly reduced in future experiments by adding additional steel collimation layers directly in front of the last HDPE layers and a cadmium layer around the detector can be used to filter out thermal neutrons. In addition, a lead shielding around the detector can reduce the gamma contribution.

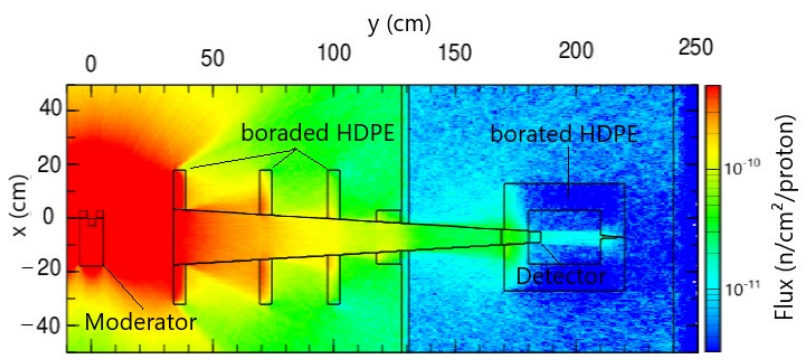

Fig. 8. Simulated flux of neutrons with energies between 1 and $10 \mathrm{eV}$. Neutrons are collimated by four layers of borated HDPE and enter the detector shielding through a tunnel. The signal to background ratio inside the detector shielding is (15 \pm 4$): 1$. 


\section{Experimental Results}

\subsection{Determination of the Signal to Background Ratio}

In the experimental campaign at PHELIX, two methods were used to determine the signal to noise ratio. For the first method, the thermal neutron spectrum is compared with and without a moderator. The result can be seen in Fig. 9. The ratio between these shots is $(16 \pm 1): 1$ for thermal neutrons and $(6 \pm 1): 1$ for epi-thermal neutrons at $1 \mathrm{eV}$. The lower ratio for faster neutrons is linked to the contribution of thermalized background neutrons which are generated in the shielding and their arrival time coincides with epi-thermal neutrons from the moderator.

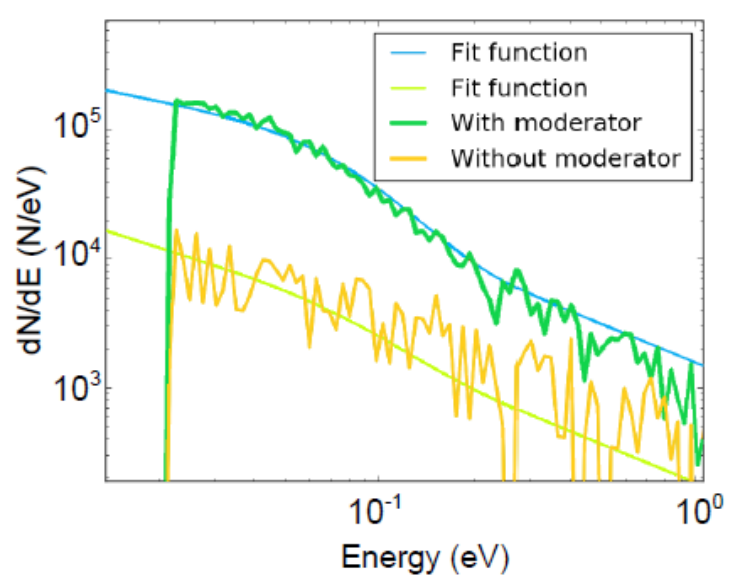

Fig. 9. Measurement of a thermal neutron distribution of a laser-based NRS campaign at PHELIX. Orange lines represent data taken without a moderator and green lines are single shot spectra with the moderator in place.

The second method used is the introduction of a black resonance filter in the beam path. ${ }^{113} \mathrm{Cd}$ has a giant resonance below $1 \mathrm{eV}$ with cross sections above $10^{4} \mathrm{~b}$ and is consequently opaque for thermal neutrons. A 1 $\mathrm{mm}$ thick cadmium plate is, therefore, placed in front of the entrance of the last collimator tunnel to block the direct line of sight to the moderator. The result can be seen in Fig. 10 together with the cadmium cross section in red and a shot without the Cd filter, indicated by the blue line. The ratio between these two shots at $25 \mathrm{meV}$ is $(17 \pm 1): 1$ which is in good agreement to the first method with $(16 \pm 1): 1$ as well as with the simulated result of $(15 \pm 4): 1$.

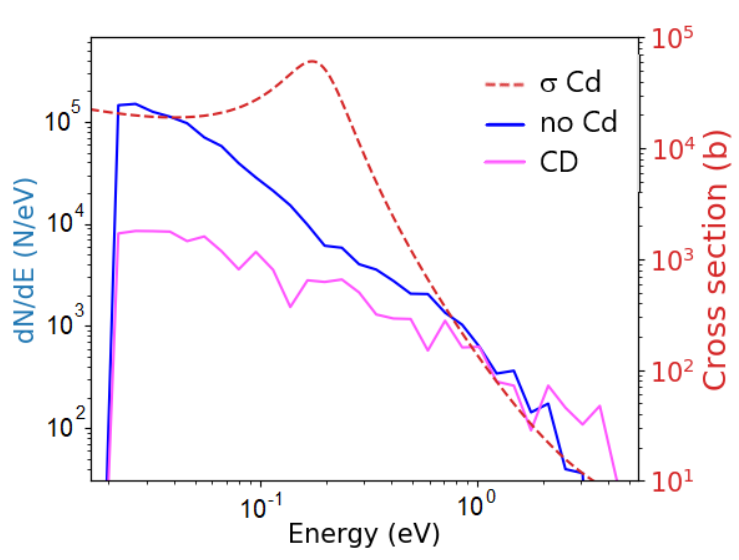

Fig. 10. Blue line: Moderated neutron spectrum without a sample. Pink line: Neutron spectrum with a $1 \mathrm{~mm}$ thick $\mathrm{Cd}$ sample in the beam path as black resonance filter. Red dashed line: The cross section of ${ }^{113} \mathrm{Cd}$. With increasing $\mathrm{Cd}$ cross section, the blue and red curve start to deviate until cadmium is opaque and all contributions in the pink graph are from the background.

\subsection{Characterization of the Moderated Neutron Spectrum}

The moderated neutron spectrum consists of two parts. The first one is a Maxwellian distribution of thermalized neutrons with the temperature of the moderator. The second part is the result of stochastical collisions and energy transfer and it follows a $1 / E^{b}$ distribution where $\mathrm{E}$ is the neutron kinetic energy and $\mathrm{b}$ is the leakage factor. $b$ describes how many neutrons are either leaked out of the moderator or have been absorbed during the moderation process. An infinite moderator without absorption would have $b=1$. In Fig. 11 the moderated neutron spectrum is compared to a simulated neutron spectrum.

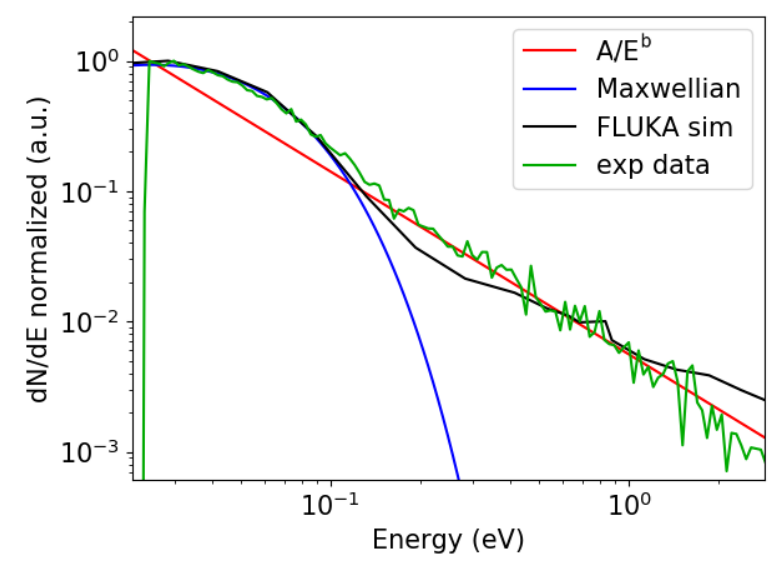

Fig. 11. Normalized experimental neutron spectrum (green) with a Maxwellian fit (blue) and $\mathrm{A} / E^{b}$ in red. The important fit parameters are $T=291 \mathrm{~K}$ and $b=1.4$. The black line represents the corresponding FLUKA simulation with $T=289 \mathrm{~K}$ and $b=$ 0.92 .

For the experimental data, a Maxwellian distribution can be fitted to the thermal peak with a temperature $T=$ $(291 \pm 15) \mathrm{K}=(18 \pm 15)^{\circ} \mathrm{C}$. This temperature is close to the room temperature of the PHELIX laser bay with $20^{\circ} \mathrm{C}$. The leakage coefficient $b$ on the other hand corresponds 
to a value of 1.4. This is not realistic since $b \leq 1$ has to be fulfilled for every moderator [15]. A FLUKA [16] simulation with a matched ion source spectrum for this experiment produces a leakage coefficient of $b=0.92$. This discrepancy suggests that the used detector is less sensitive for fast neutrons than the $1 / E^{0.5}$ scaling of the boron cross section would suggest. This is most likely caused by an oversaturation of the MCP during the $\gamma$-flash from the laser target interaction. For future applications, it is therefore advisable, to shield the direct line of sight between target and diagnostic with a high $\mathrm{Z}$ material.

\section{Conclusion}

The proposed laser neutron setup was developed and optimized to identify materials through the detection of nuclear resonances via NRS. The optimization was done with PHITS simulations for a setup at the PHELIX laser facility in Darmstadt. The setup was characterized with simulations and validated with an experiment, which was in agreement with the predictions. The catcher shielding, the moderator as well as the collimation system show large improvements to previous laser-driven neutron spectroscopy experiments and can be used to demonstrate the capability to detect various isotopes inside a sample.

\section{Acknowledgement}

The authors want to especially thank the PHELIX laser team at GSI for their work on the realization of this experimental campaign as well as the GSI Plasma Physics Department. This work is supported by the LOEWE excellence initiative of the state of Hessen.

\section{References}

1. R. Nelson, O. Ronald, et al. Journal of Imaging 4.2 : 45 (2018)

2. Z. Révay, et al., Handbook of nuclear chemistry: 1619 (2011)

3. P. Schillebeeckx, et al., Springer, I/24. 10-66. (2015)

4. Nuclear research and industry today. Nat Rev Phys 1, 171 (2019)

5. M. Roth, et al., Phys. rev. lett. 110.4044802 (2013)

6. B. Rus, et al., Vol. 8780. International Society for Optics and Photonics (2013)

7. E. Mauerhofer, et al., No. FZJ-2017-07855. Streumethoden, (2017)

8. S. F. Nowicki, et al., Physics Procedia 90: 374-380 (2017)

9. V. Bagnoud, et al., App. Phys. B 100.1 137-150 (2010)

10. R. A. Snavely, et al., PRL, 85.14: 2945. (2000)

11. F. Wagner, et al., Phys. rev. let. 116. 20 (2016)

12. A. Kleinschmidt, et al. PoP. 25.5053101 (2018)
13. A. J. Koning, et al., Nuc. data sheets 113.12: 28412934 (2012)

14. P. Tsai, et al., Nuc. Inst. and Meth. in Phys. 416: 1629. (2018)

15. I. Petrovic, P. Benoist, Adv. in Nuc. Sci. and Tec. Springer, Boston, MA, (2002)

16. G. Battistoni, et al., AIP Conference proceedings. Vol. 896. No. 1. Am. Inst. of Phys., (2007) 